\title{
A Data Consistent Variational Segmentation Approach Suitable for Real-time Tomography
}

\author{
Henri Der Sarkissian*, Nicola Viganò, Kees Joost Batenburg ${ }^{\dagger}$ \\ Centrum Wiskunde en Informatica, Computational Imaging group \\ Science Park 123 \\ 1098 XG Amsterdam, The Netherlands \\ \{henri.der.sarkissian, nicola.vigano, joost.batenburg\}@cwi.nl
}

\begin{abstract}
Computed Tomography (CT) is an imaging technique that allows to reconstruct volumetric information of the analyzed objects from their projections. The most popular reconstruction technique is the Filtered Back Projection (FBP). It has the advantage of being the fastest technique available, but also the disadvantage to require a high number of projections to retrieve good quality reconstructions. In this article we propose a segmentation method for tomographic volumes composed of few materials. Our method combines existing high-quality variational segmentation frameworks with the data consistency approach used in tomography and discrete tomography. We show that our algorithm performs well under high noise level and with moderately low number of projections, and that the data consistency significantly improves the segmentation, at the cost of only one FBP reconstruction and forward projection.
\end{abstract}

\section{Introduction}

Computed Tomography (CT) is an imaging technique that allows to reconstruct volumetric information of the analyzed objects from their projections. The most popular reconstruction technique is the Filtered Back Projection (FBP) [19]. It has the advantage of being the fastest technique available, but also the disadvantage to require a high number of projections to retrieve good quality reconstructions. Iterative algorithms like the Simultaneous Iterative Reconstruction Technique (SIRT) allow to reduce

\footnotetext{
*Address for correspondence: Centrum Wiskunde en Informatica, Science Park 123, 1098 XG Amsterdam, The Netherlands.
}

${ }^{\dagger}$ Also affiliated at: Leiden University, Department of Mathematics, 2300 RA Leiden, The Netherlands 
the amount of required projections, at the expense of a greater reconstruction time [17]. Along the same lines of the SIRT algorithm, more advanced algorithms that impose strong priors on the reconstruction allow to reconstruct images from extremely low numbers of projections, but they also require even longer times to reconstruct.

To extract physical and morphological information about the analyzed samples, it is often necessary to identify and segment regions and features inside the reconstructed volumes. This process allows to distinguish them from the background and other features present in the volume. The segmentation process can be simply understood in terms of a classification problem, where the unit volumes of the reconstructed object (voxels) have to be assigned to different regions. The simplest segmentation approach is the intensity thresholding method, where voxels are segmented on the basis of their reconstructed intensity. Their intensity determines, with respect to the user provided thresholds, to which region of the volume they belong to. Some of the more advanced reconstruction algorithms, which use homogeneity of the reconstructed objects as prior, intrinsically deliver segmented reconstructions, on top of having the low requirements for input data that they are known for. Typical examples of such algorithms include the discrete tomography algorithms DART [5] and TVR-DART [44].

Fast processing of tomographic data is becoming increasingly important in recent times, because it is of fundamental importance for the real-time observation of evolving processes, as they happen. This includes the volume reconstruction and feature segmentation inside the reconstructed volume. The requirements imposed by real-time applications, conflict with most of the solutions proposed so far. These methods either sacrifice acquisition speed for fast processing, for instance in the case of the joint FBP reconstruction and simple thresholding approach, or they sacrifice the data analysis speed, in exchange for faster acquisitions like in the case of the discrete tomography algorithms.

In this article we propose a feature segmentation scheme that only requires two FBP operations and can work with moderately low number of projections.

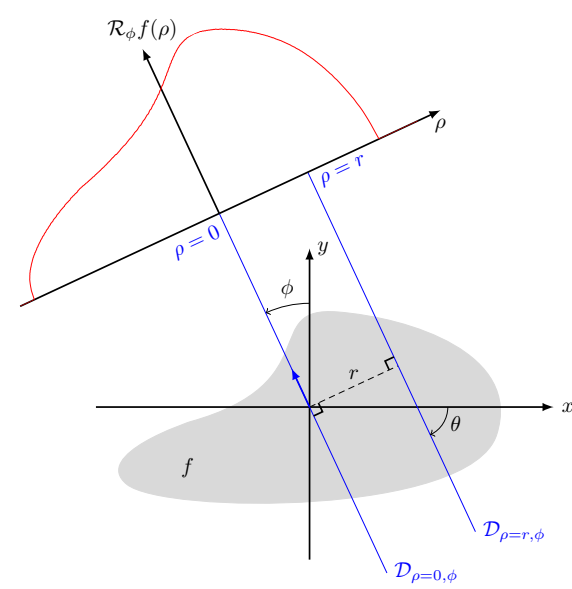

Figure 1: Two-dimensional tomography setup 


\subsection{Tomography setup}

In tomography, projections of a volume are usually acquired by means of a penetrating beam (e.g. $\mathrm{X}$-rays, electron beam, etc) and they correspond to collections of line integrals throughout the volume. The tomographic reconstruction is classified as an inverse problem, and it is the process of recovering a volume from its projections. Figure 1 depicts a typical tomographic setup in 2D. In this paper, we refer to the tomographic reconstruction problem as the solution to a linear system. This formulation remains general and covers $2 \mathrm{D} / 3 \mathrm{D} / n \mathrm{D}$ cases as well as any kind of geometry and beam orientation.

The reconstruction volume is often discretized into identical unit volumes called voxels, while the projections are the collection of detector pixel values. The volume and the projections are represented as vectors $\boldsymbol{v} \in \mathbb{R}^{n}$ and $\boldsymbol{p} \in \mathbb{R}^{m}$ respectively. We denote $\boldsymbol{W} \in \mathbb{R}^{n \times m}$ the projection sensing matrix, which corresponds to the relationship between the volume and detector spaces. As a result, the forward model of a tomographic acquisition is represented by the following:

$$
\boldsymbol{p}=\boldsymbol{W} \boldsymbol{v} .
$$

The Filtered Back-Projection [19] algorithm (or FDK in cone-beam geometry [15]) inverts eq. (1) by considering the geometrical and spectral relations of the projections in a one-step approach

$$
\boldsymbol{x}=\boldsymbol{W}^{\top} \mathcal{F} \boldsymbol{p},
$$

where $\mathcal{F}$ is a high-pass filter and $\boldsymbol{W}^{\top}$ is the adjoint operation to the projection called back-projection.

\subsection{Related work}

\subsubsection{Segmentation}

In a computer vision perspective, segmentation is performed on the characteristics of the reconstructed volume. Simple approaches are based on the grey-value histogram of the volume $[25,31]$ and region statistics [43]. More recent and attractive techniques are based on functional optimization. These techniques are gaining popularity thanks to their ability to incorporate model constraints and regularity criteria. Some notable examples are: curvature or boundary length in the famous Mumford-Shah functional [22], and shape priors [29, 11]. The solution of these variational models can be achieved in a continuous representation by parametric curve evolution algorithms [8], level-sets methods [24, 23, 13, $36,7]$ and hybrid approaches [1]. In line with the levelsets methods, convex relaxation techniques are also fast and stable alternative [12,9]. Finally, graph-based techniques are the discrete counterparts of the just mentioned variational techniques. They aim at optimizing the segmentation functional directly on the discrete grid on which the volumes are expressed by maximum flow/minimal cut techniques. Popular frameworks include graph-cut [6] and more general formulations like random walker [16] and power watersheds [14].

\subsubsection{Discrete tomography}

The previously described computer vision approaches tend to consider the reconstructed volume alone, ignoring the characteristics of the system on which the data have been produced. Discrete tomography 
is a special case of tomography where the representation of the reconstructed object is assumed to be composed of only a few different intensity values. This constitutes a strong reconstruction prior, which guarantees higher quality reconstructions when it holds true. There are many links between discrete tomography and segmentation since a large number of proposed methods consider either segmentation as part of the reconstruction process or impose a segmented reconstruction by the means of priors. Discrete tomography ramifications closely resemble the segmentation taxonomy. Hence, the DART algorithm [5] alternates between an algebraic reconstruction step and a thresholding step, TVR-DART [44] is based on the same thresholding step augmented by a TV regularization under a variational approach. Other modern methods include levelset-based techniques using the MumfordShah functional [40, 27, 42, 37, 38] or parametric curve approaches [1, 2], convex approaches [33], and graph-based approaches [20, 32].

\subsubsection{Hybrid tomography oriented segmentation}

The two presented approaches differ significantly in their computational requirements. The image driven segmentation methods are generally very fast. On the other hand, discrete tomography is a hard and costly problem, and the algorithms to solve it can be significantly slower than the segmentation counterparts.

Hybrid approaches to segmentation of tomographic images attempt to use the projection data as prior knowledge to guide the segmentation process in the image domain [4, 3]. These approaches aim at minimizing the re-projection distance, which is the distance between the measured projections and the projected segmented image. Although they show superior performance over classical thresholding schemes, having much in common with thresholding algorithms, they are sensitive to reconstruction artifacts and noise in the data. Moreover, due to the discontinuity of the hard thresholding operator, these techniques rely on heuristic and slowly converging solvers. This problem has been alleviated recently by using a continuous and differentiable relaxation of the thresholding function [44].

The reconstructed residual error (RRE) has been proposed as a local inconsistency indicator of the segmentation quality and consistency with respect to the projection data [28]. It relies on the fact that while the unsegmented image is generally a good solution to the tomographic problem, the segmented image is generally not anymore. Hence, the reconstruction discrepancies with the perfect reconstruction mostly belong to the tomography operator null space before segmentation. After segmentation, because inconsistencies have been introduced, most of the remaining error lies in the range of the operator. Thus it can be efficiently highlighted by reconstructing the residual between the reprojection of the segmented volume and the initial projections [28].

\subsection{Outline of the paper}

In this paper, we propose an approach based on hybrid methods, with the goal of producing a tomography consistent segmentation of tomographic volumes without the computational burden of iterative reconstruction. In this perspective, the use of forward- and back-projection operations is limited to the reconstruction of the projection residual error. 
The paper is organized as follows. First, we describe the proposed two steps segmentation approach. Then, we propose a refinement model based on the reconstructed residual error as a segmentation confidence measure. Finally, we present and discuss numerical examples.

\section{Proposed approach}

In the previous section we introduced the classical volume based segmentation methods, which have the advantage to be fast but incorporating prior knowledge from the tomographic problem. We also saw that they may lead to heavy but more accurate discrete tomography iterations. Here we propose a strategy to combine both methods and build a segmentation model of $K$ piecewise constant regions for tomographic images. Hence, we start by getting a first segmentation using only the reconstructed data from a fast analytical reconstruction. Then segmentation inconsistencies with respect to the projection data are computed and used to refine the initial segmentation. This process is sketched in fig. 2.

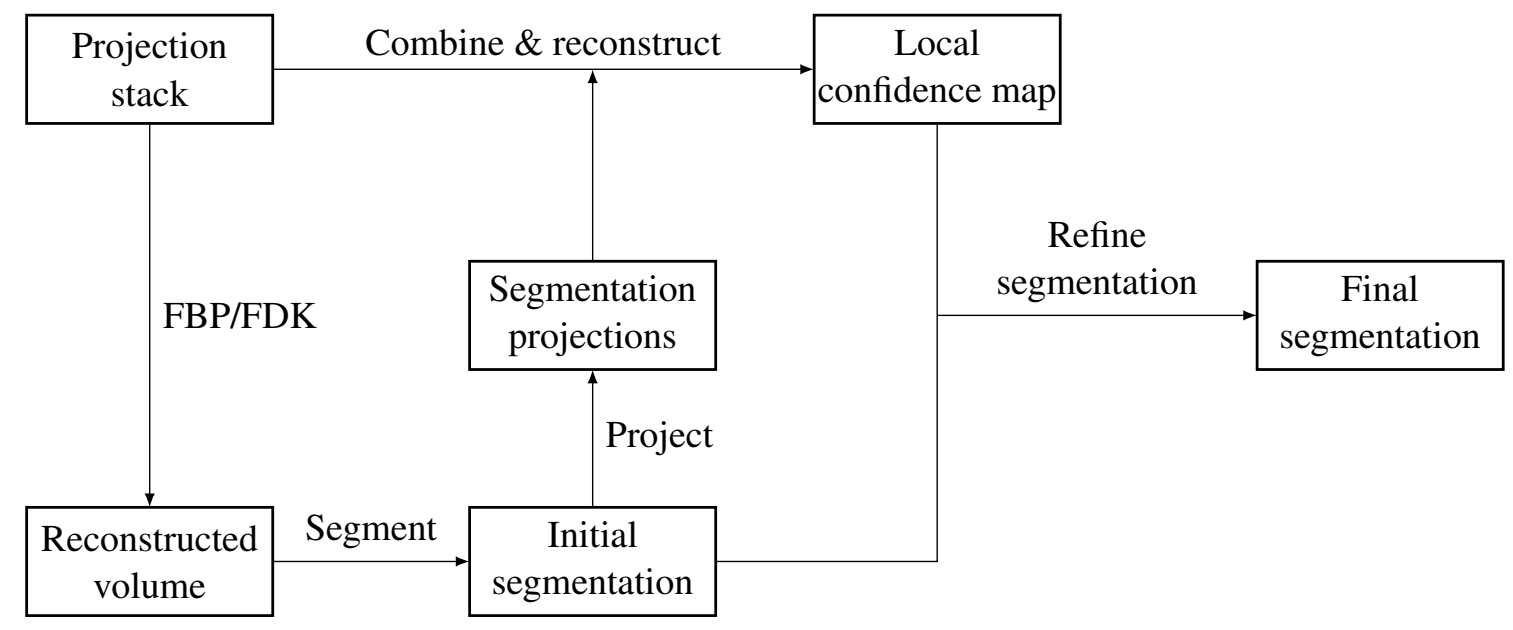

Figure 2: Steps of the proposed algorithm

\subsection{Initial variational segmentation}

Let $f$ denote a $d$ dimensional reconstructed tomographic volume. A piecewise constant model of $f$ defined on an open domain $\Omega \subset \mathbb{R}^{d}$ is

$$
\begin{aligned}
f: \Omega & \rightarrow\left\{\rho_{1}, \ldots, \rho_{K}\right\} \\
\Omega_{i} & \mapsto \rho_{i}
\end{aligned}
$$

where $\Omega_{1}, \ldots, \Omega_{K}$ form a partition of $\Omega$. The segmentation problem is the determination of the disjoint regions $\Omega_{i}$ as well as the optimal values $\rho_{i}$.

Variational methods recast an inverse problem into an optimization problem, and they define optimality conditions for the segmentation problem by means of $n$ energy functionals. The solution is 
represented by the minimizer of the sum of such such energy functionals. One common choice of energy definition is the Mumford-Shah functional. In theory, its minimization results in the optimal partitioning of the input volume [22].

\subsubsection{Binary segmentation}

The binary or two-phase segmentation is the partitioning case where $K=2$. The piecewise-constant model of the Mumford-Shah segmentation functional is expressed as:

$$
\underset{c_{1}, c_{2}, \Omega_{1}}{\arg \min } E\left(c_{1}, c_{2}, \Omega_{1}\right)=\mu\left(\int_{\Omega_{1}}\left|f(x)-c_{1}\right|^{2} d x+\int_{\Omega \backslash \Omega_{1}}\left|f(x)-c_{2}\right|^{2} d x\right)+\lambda \int_{\partial \Omega_{1}} d s,
$$

where $\lambda$ and $\mu$ are constant model parameters and the right most term denotes the length of the segmented region contour. This equation corresponds to the popular Chan and Vese model [13]. We use a convex relaxation formulation of this problem defined as:

$$
\underset{c_{1}, c_{2}, u: \Omega \rightarrow\{0,1\}}{\arg \min } E\left(c_{1}, c_{2}, u\right)=\mu \int_{\Omega}\left[\left|f-c_{1}\right|^{2} u+\left|f-c_{2}\right|^{2}(1-u)\right]+\lambda \int_{\Omega}|\nabla u| .
$$

The boundary length corresponds to the total variation of the indicator function $u$. This problem can be solved globally, through simultaneous estimation of the region intensities and the region shape. However, in practice a two step minimization procedure is preferred:

- for a fixed segmentation $u$, the optimal values of $c_{1}$ and $c_{2}$ are easily computed in a closed form as the mean intensity within each region

$$
\left\{\begin{array}{l}
c_{1}=\frac{\int_{\Omega} f(x) u(x) d x}{\int_{\Omega} u(x) d x} \\
c_{2}=\frac{\int_{\Omega} f(x)(1-u(x)) d x}{\int_{\Omega}(1-u(x)) d x}
\end{array}\right.
$$

- for fixed $c_{1}$ and $c_{2}$, the functional is convex with respect to $u$.

Finally, the binary set $\{0,1\}$ is non-convex. We can relax this constraint by performing the optimization over the convex hull $[0,1]$ and thresholding the solution afterwards. This convex relaxation approach has been proved to converge to a global minimum of the binary energy [12]. The corresponding convex problem is expressed as:

$$
\min _{c_{1}, c_{2}, \boldsymbol{u} \in[0,1]^{n}} \sum_{i=1}^{n}\left(\boldsymbol{f}_{i}-c_{2}\right)^{2} \boldsymbol{u}_{i}+\sum_{i=1}^{n}\left(\boldsymbol{f}_{i}-c_{1}\right)^{2}\left(1-\boldsymbol{u}_{i}\right)+\alpha \sum_{i=1}^{n}\|\nabla \boldsymbol{u}\| .
$$

We used the deriving instance of Chambolle and Pock's primal-dual algorithm [10] to efficiently optimize the segmentation functional with total variation regularization and box constraints. 


\subsubsection{Multi-level segmentation}

We now consider the case with more than one object with different attenuation properties, i.e. $K>$ 2. In this case, even though convex relaxations of the problem have been investigated, no global optimality convergence have been proved. Classically, the problem is tackled by using multiple levelset functions [36, 9].

In our case, we consider the following formulation, which only requires the optimization of a multivalued function $u$

$$
\min _{c_{1}, \ldots, c_{n}, \boldsymbol{u} \in \mathbb{R}^{n}} \frac{1}{2} \sum_{k=1}^{K} \sum_{i=1}^{n} \frac{1}{\left|F(i)-c_{k}\right|^{2}}\left|u(i)-c_{k}\right|+\alpha \sum_{i=1}^{n}\|\nabla u\| .
$$

As in the binary case, Chambolle and Pock's algorithm is used for optimization of the multilevel segmentation functional. As in the binary case, a threshold was finally applied between the target intensities $c_{k}$.

\subsection{Refinement of the segmentation guided by tomographic data}

In this section, we detail the refinement procedure. The idea is to modify the segmentation towards consistency with the projection data. We proceed in two steps. First, a local segmentation confidence map is computed using the projection data. Then, this confidence map is used to refine the segmentation in a variational model.

\subsubsection{Estimation of the segmentation inconsistencies using the projection data}

The unsegmented image resides in the solution space of the tomographic problem, while the segmented image usually does not anymore [28]. It presents inconsistencies, which can be measured with either the reprojection distance [4] or the projection stack inconsistency [34]. For this reason,

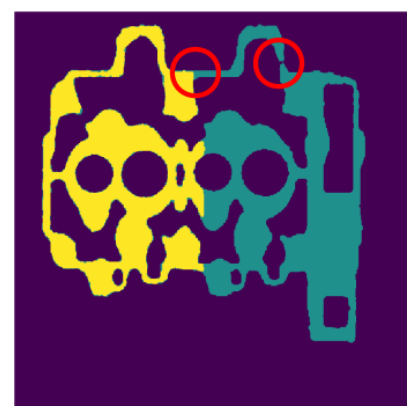

(a)

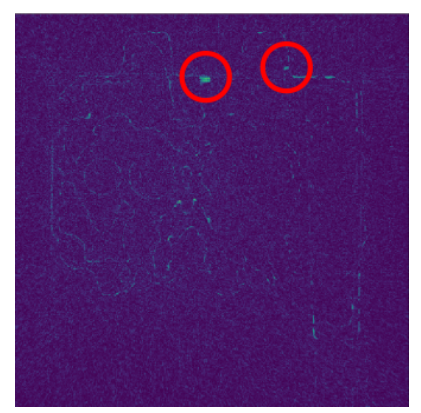

(b)

Figure 3: Visualization of the segmentation inconsistencies with the reconstructed residual error. (a) Multilabel segmentation with two inconsistencies highlighted in the red circles. (b) Reconstruction of the residual error with a filtered backprojection. The segmentation inconsistencies are clearly visible in the residual reconstructed error. 
the reconstructed residual error (RRE) has been proposed as a local (in)consistency indicator [28]. Figure 3 shows an example of segmentation (fig. 3a) and the resulting RRE (fig. 3b). We can notice the correlation between the actual misclassified regions and the residual reconstructed error.

Let $s$ be the segmentation at this point with estimated grey-values. The reconstructed residual error is computed as:

$$
\boldsymbol{r}=\boldsymbol{W}^{\dagger}(\boldsymbol{W} \boldsymbol{s}-\boldsymbol{p})
$$

where $\boldsymbol{W}^{\dagger}$ denotes the pseudo-inverse of the projection matrix. In practice, the projection matrix is too large to allow its pseudo-inverse to be computed explicitly, and $\boldsymbol{W}^{\dagger}$ is approximated by a reconstruction algorithm [28]. For the sake of keeping the computational complexity low, we use the FBP algorithm.

\subsubsection{Estimation of grey levels}

In the previous segmentation step, flat grey values in the image (the constants $c_{i}$ ) were either provided or optimized in the segmented procedure. With poor reconstruction quality, significant discrepancies can arise between the reconstructed levels and the actual attenuation coefficients of the materials. These model parameters optimize the least-square projection distance criterion:

$$
\|\boldsymbol{W} \boldsymbol{s}-\boldsymbol{p}\|_{2}^{2}=\left\|\boldsymbol{W} \sum \mu_{k} \delta\left(\boldsymbol{s}-c_{k} \mathbf{1}\right)-\boldsymbol{p}\right\|_{2}^{2} .
$$

In fact, eq. (10) is quadratic in $\boldsymbol{\mu}$ and yields a closed form solution [4]. Let us define for that purpose the system matrix $\boldsymbol{A} \in \mathbb{R}^{N_{\text {proj }} \times K}$ :

$$
\boldsymbol{A}=\boldsymbol{W}\left(\begin{array}{ccc}
\mid & \mid & \mid \\
\delta\left(s-c_{1} \mathbf{1}\right) & \cdots & \delta\left(s-c_{K} \mathbf{1}\right) \\
\mid & \mid & \mid
\end{array}\right)
$$

Solving eq. (10) is equivalent to finding the least-square solution of $\boldsymbol{A} \boldsymbol{\mu}=\boldsymbol{p}$, leading to the resolution of the $K \times K$ normal equations system $\boldsymbol{A}^{\top} \boldsymbol{A} \boldsymbol{\mu}=\boldsymbol{A}^{\top} \boldsymbol{p}$.

\subsubsection{Segmentation refinement}

The reconstructed residual error is locally large when the segmentation is inconsistent with the projection data and low otherwise. We want the regions with high error being able to switch their labels while low error regions might remain untouched. Hence, the segmentation refinement problem is finally expressed as a weighted ROF problem [30]

$$
\boldsymbol{s}_{\boldsymbol{C}}=\underset{\boldsymbol{x}}{\arg \min }\|\boldsymbol{C}(\boldsymbol{x}-\boldsymbol{s})\|_{2}+\lambda\|\boldsymbol{x}\|_{\mathrm{TV}}
$$

The weighting matrix $\boldsymbol{C}$ is a diagonal matrix indicating the confidence level of the segmentation for every voxel. A low confidence level would locally favour label continuity within the neighborhood while a high intensity is according more weight to the data fidelity term. This approach can thus be seen as a locally constrained total variation problem [39]. 
We define the local segmentation confidence map from the reconstructed residual error $\boldsymbol{r}$ by the relation $\boldsymbol{C}_{i, i}=g\left(\boldsymbol{r}_{i}\right)$ where

$$
g(\cdot)=\frac{1}{1+|\cdot|^{\beta}}
$$

or

$$
g(\cdot)=\exp (-\beta|\cdot|)
$$

The choice of the function $g$ is inspired by anisotropic diffusion [26, 41] since, in a way, we want the region labels to diffuse between the segmented regions under spatial constraints. Algorithm 1 summarizes the refinement process.

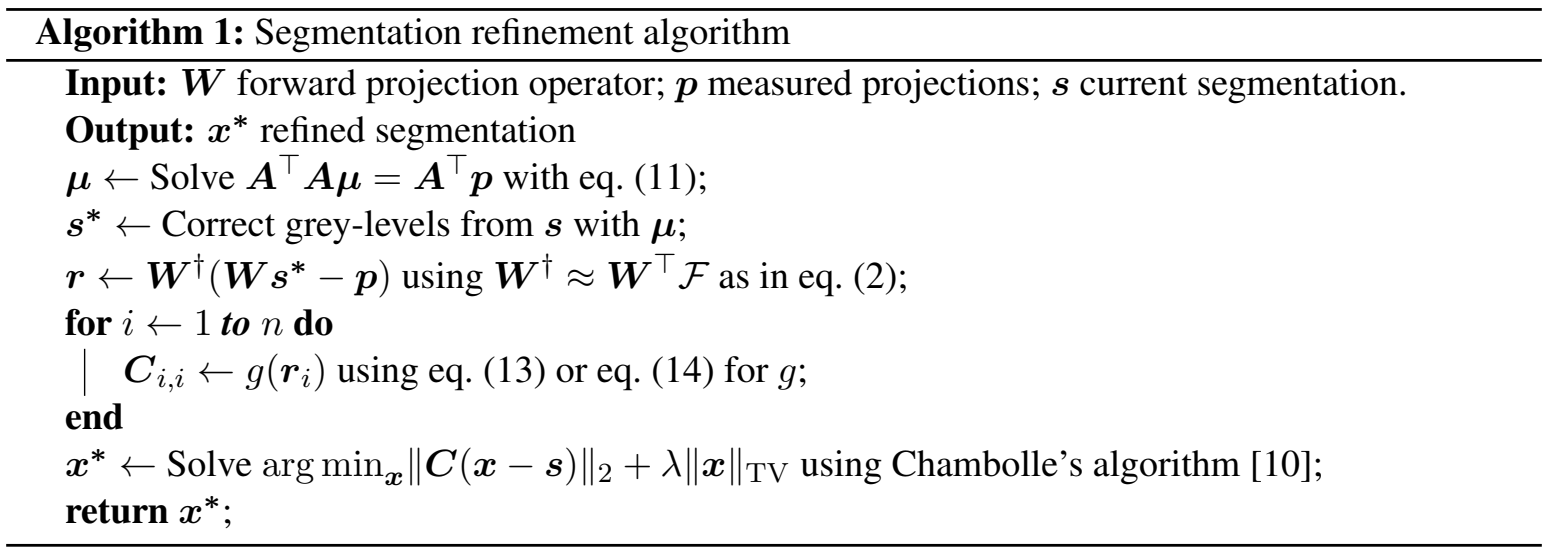

\subsection{Computational considerations}

Having described the method, we proceed here to compare its computational performance with respect to other practical computational approaches. We derive the computational complexity for the twodimensional version of each method, starting from their composing steps.

Let us consider a $n \times n$ reconstruction grid (the vector $\boldsymbol{x}$ ), and for $p$ projections (the vector $\boldsymbol{p}$ ) with $n$ bins per projection. A simple back-projection and forward-projection have a complexity of $O\left(n^{2} p\right)$ each, while the filtering step of FBP has a complexity of $O\left(n^{2}\right)$ per projection when computed in direct space, and $O(n \log n)$ when computed in Fourier space. A simple thresholding step has complexity of $O\left(n^{2}\right)$ because it is performs a single iteration through all the reconstruction voxels, while an iterative denoising step has complexity in the order of $O\left(n^{2} t_{\mathrm{D}}\right)$ where $t_{\mathrm{D}}$ is the number of iterations of the denoising operation. The solution to the problems in equations eq. (7) and eq. (12) have similar complexities to the denoising step: $O\left(n^{2} t_{\mathrm{LS}}\right)$ and $O\left(n^{2} t_{\mathrm{R}}\right)$ respectively, where $t_{\mathrm{LS}}$ and $t_{\mathrm{R}}$ are the number of iterations performed to solve each problem. Here we assume that $t_{\mathrm{D}}, t_{\mathrm{LS}}$ and $t_{\mathrm{R}}$ are chosen to be the same and equal to $t$.

An FBP reconstruction followed by a simple thresholding step, requires a filtering step, a backprojection and simple thresholding, resulting in the worst case in a complexity of $O\left(n^{2} p+n^{2} p+n^{2}\right)=$ $O\left(n^{2}(2 p+1)\right)=O\left(n^{2} p\right)$. An FBP reconstruction followed by a denoising step and a thresholding step, 
only adds the denoising step to the previous calculation, resulting in a complexity of $O\left(n^{2} p+n^{2} p+\right.$ $\left.n^{2} t_{D}+n^{2}\right)=O\left(n^{2}\left(2 p+t_{D}+1\right)\right)=O\left(n^{2}(p+t)\right)$. The method proposed in this article substitutes the denoising step with solutions to the problems in equations eq. (7) and eq. (12), a forward-projection and an additional FBP. This translates in a complexity of $O\left(n^{2} p+n^{2} p+n^{2} t_{\mathrm{LS}}+n^{2} p+n^{2} p+n^{2} p+\right.$ $\left.n^{2} t_{\mathrm{R}}+n^{2}\right)$, where we assume again that $t_{\mathrm{LS}}=t_{\mathrm{R}}=t$, resulting in a complexity of $O\left(n^{2}(5 p+\right.$ $2 t+1))=O\left(n^{2}(p+t)\right)$. The number of iterations $t$ is usually fixed, and the complexity of the $O(p+t)$ is then identical to the complexity of the term $O(p)$. This means that from a computational perspective, the proposed method is in the same ballpark of simpler methods like FBP followed by a simple thresholding.

The SIRT algorithm performs as many forward-projections and back-projections as prescribed iterations, plus a volume update at each iteration. This translates in a complexity of $O\left(2 t_{\mathrm{SIRT}} n^{2} p+\right.$ $t_{\text {SIRT }} n^{2}$, where $t_{\text {SIRT }}$ is the number of SIRT iterations. For a SIRT reconstruction followed by simple thresholding we have a complexity of $O\left(2 t_{\mathrm{SIRT}} n^{2} p+t_{\mathrm{SIRT}} n^{2}+n^{2}\right)=O\left(t_{\mathrm{SIRT}} n^{2} p\right)$, while for a SIRT reconstruction followed by a denoising step and simple thresholdding we have a complexity of $O\left(2 t_{\mathrm{SIRT}} n^{2} p+t_{\mathrm{SIRT}} n^{2}+n^{2} t_{D}+n^{2}\right)=O\left(n^{2}\left(t_{\mathrm{SIRT}} p+t_{\mathrm{D}}\right)\right)$. Finally, for a DART iteration, we perform a certain number of SIRT iterations on the contour of the reconstruction region. In the worst case, this translates in a complexity of $O\left(t_{\text {DART }}\left(2 t_{\text {SIRT }} n^{2} p+t_{\text {SIRT }} n^{2}\right)+n^{2}\right)=O\left(t_{\text {DART }} t_{\text {SIRT }} n^{2} p\right)$. This shows that more advanced methods like the SIRT and DART algorithms have typically larger complexities than the proposed method.

\section{Numerical examples}

We now test the performance of the proposed approach with a few selected cases. For each experiment, projections have been simulated with the ASTRA toolbox [35]. Through this section, we consider only a 2D parallel-beam geometry. Different geometries could be used as well without any impact on the general methodology, but would have unnecessarily complicated the analysis. Each phantom volume has a $512 \times 512$ shape and each projection is an array of 512 detector bins. Moreover to reduce the effects of using the same projector to produce and reconstruct the data (inverse crime), the projections have been generated using the "linear" projector and the reconstructions were performed with the "cuda" forward- back-projector pair.

The simulated projections are been corrupted by three different noise sources:

- Poisson noise in the Beer-Lambert model with photon intensity $I_{0}=10^{5}$;

- Gaussian distributed background noise with $\sigma=0.4$;

- Random non-uniformity in the detector (also known as "Zigler" effect).

For each of the phantoms presented in fig. 4, the experiment has been repeated 10 times in order to account for random noise variability, and the average and the standard deviation of measures are considered. The results of our algorithm are compared to the ones obtained without the projection data driven refinement and to the PDM optimal thresholding algorithm which yields proven good performance in tomographic volume segmentation [4]. 


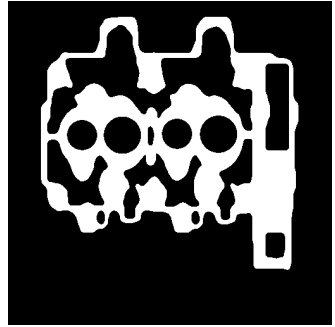

(a) Binary motor cylinders

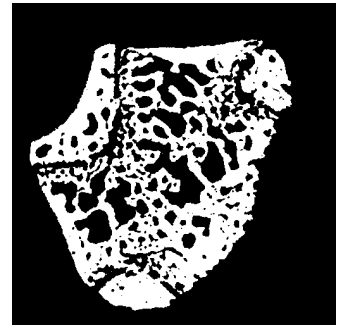

(b) Binary femur

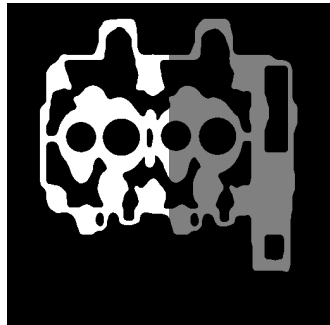

(c) Multilevel motor cylinders

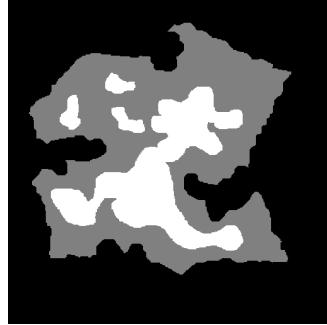

(d) Multilevel shape

Figure 4: Phantoms used in the experiment

\subsection{Binary segmentation}

We focus in this section on the binary case where the phantoms are composed of only two distinct grey levels (0 and 1).

In the first experiment, we used $\lambda=1.5$ for the first convex segmentation step and $\lambda=10^{-4}$ to $10^{-6}$ for the refinement model. The reason the two parameters are different for each task is the weighting function $g$ which shifts the relative strength of the data fidelity term in eq. (12). These parameters where found empirically and optimized with respect to the known ground-truth phantom. One important consideration for the choice of $\lambda$ is its role of balancing the data-fidelity and the regularization terms in eq. (12). Thus a good indicator for the range of $\lambda$ is the ratio between the regularizer of $f$ and the norm of weighting matrix $\|\boldsymbol{C}\|$. It is important to note, however, that more general strategies for more general datasets without known ground-truth have been studied in the literature. We refer the reader to, for example, Morozov's discrepancy principle [21], the generalized cross-validation method, and the use of L-curves [18].

Figure 5 shows the result of our segmentation algorithm on the binary motor cylinder phantom (fig. 4a). The number of projections varies for each column. We can note that the residual reconstructed error on the last row exhibits well segmentation deviations with respect to the phantom whenever artifacts are part of the segmentation, or whenever the structure boundaries are not segmented accurately.

We can also notice the behaviour of the refined segmentation guided by the RRE: the result is successfully steered towards the phantom shape. By having a closer look to the first column of fig. 5, we can see that the artifacts forming small independent structures in the initial segmentation are all successfully removed, but the rest of the object is not overly smoothed. By looking at the edges on the right side and the top side of the object, we can notice that the refined segmentation boundaries are significantly closer to the optimum. As can be seen in the zooms on fig. 5, the algorithm can also reconnect missing edges in a small extent.

Comparative quantitative results are provided in fig. 6. The proposed refinement procedure significantly lowers the misclassification error. When enough projections have been used, all the methods produce comparable results, but the refined segmentation procedure can improve over the rNMP by at 

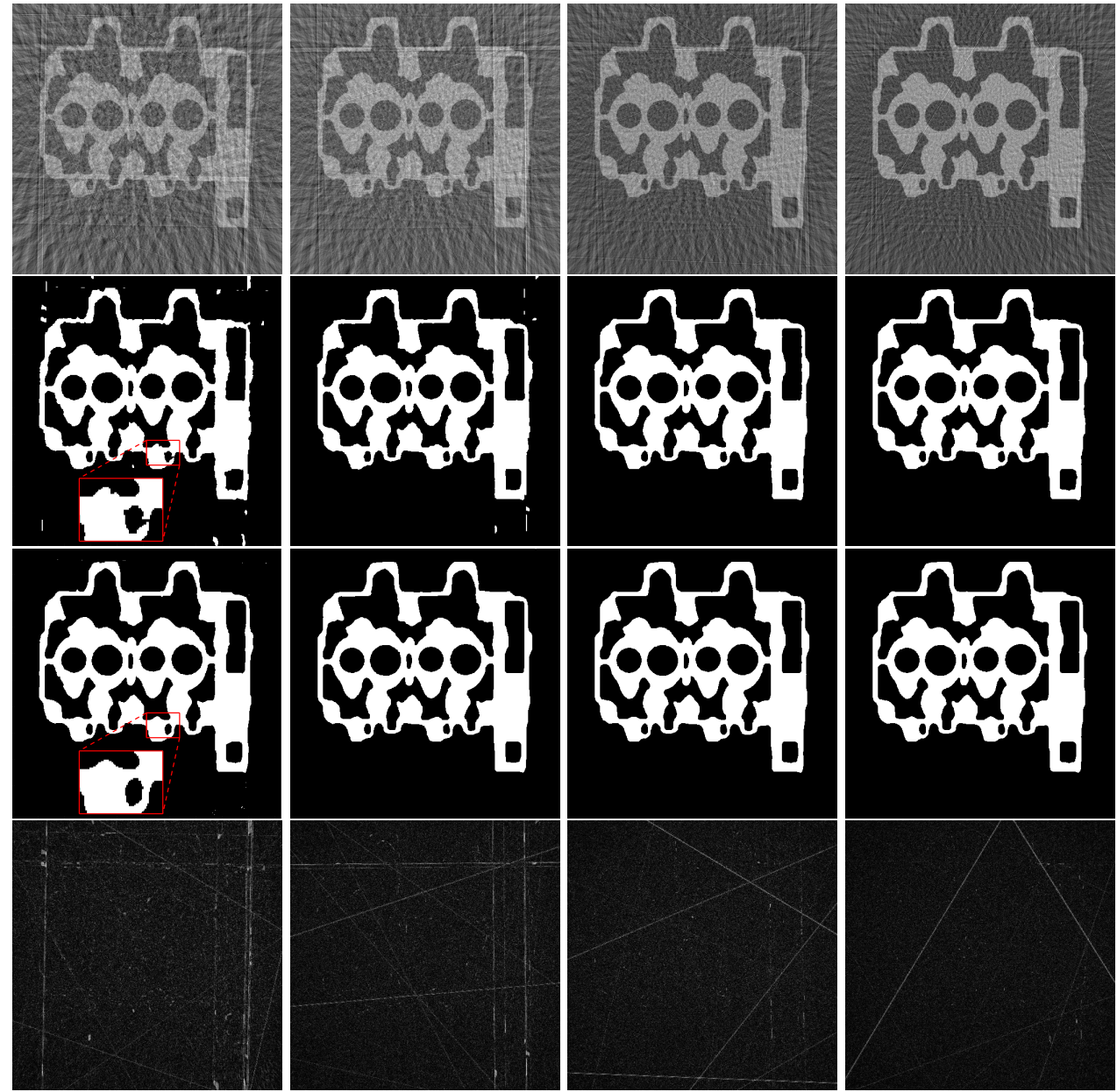

Figure 5: Binary cylinder phantom experiment. Each column is obtained by using respectively 30, 40, 50 and 60 projections. First row: Filtered backprojection reconstruction from noisy projections. Second row: Segmentation using convex formulation. Third row: Refined segmentation using our confidence map method. Fourth row: Reconstructed residual error obtained from $2^{\text {nd }}$ row.

least $10 \%$ over the initial segmentation. It can also be noted that due to the noisy reconstructions and artifacts, the PDM thresholding technique does not perform well in this situation. However, denoising the reconstruction first can significantly improve the result of PDM, which is still outperformed by our proposed method.

A more challenging phantom is the femur (fig. 4b) with more complex topology. We used in this case $\lambda=0.5$ for the convex segmentation, because the image is less smooth and contains a larger number of edges. The proposed method performs well in this case as well, as it can be seen in fig. 7 . 


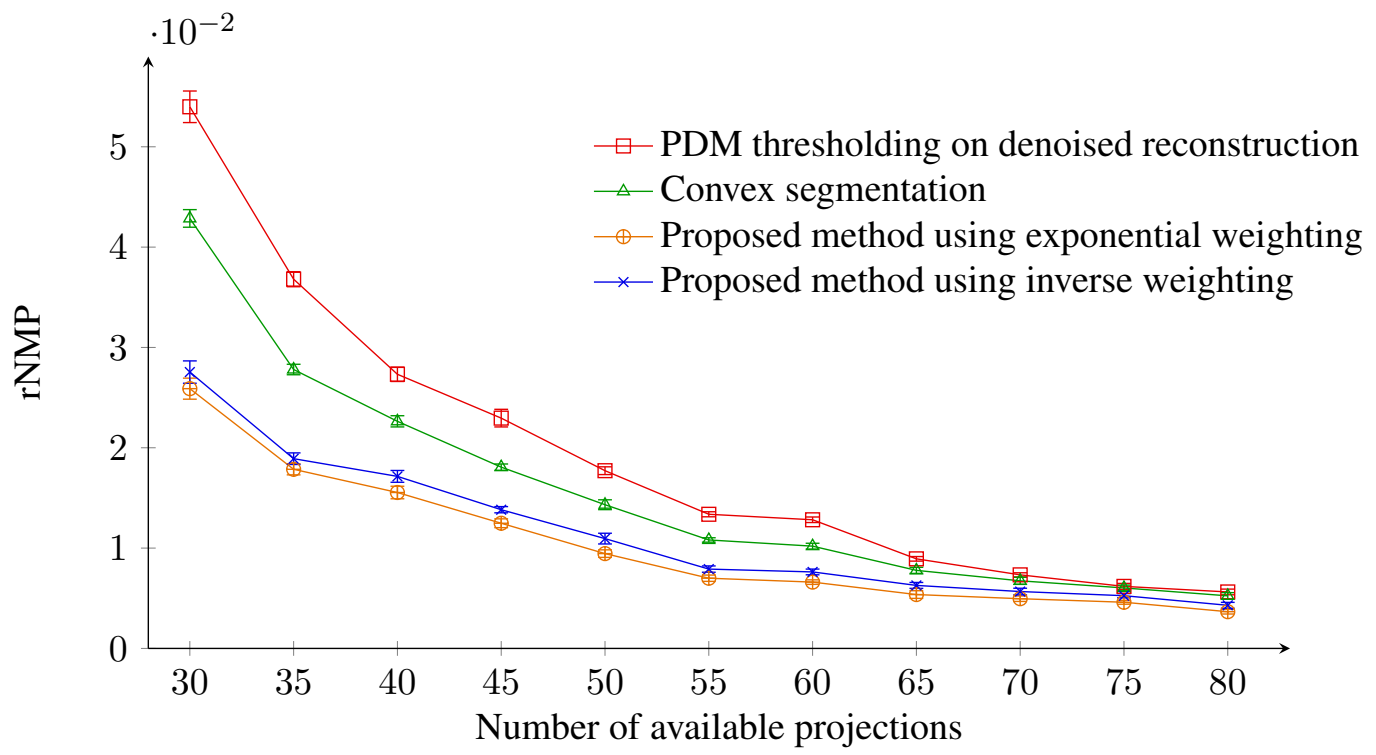

Figure 6: Segmentation error measures (rNMP) for the binary cylinder phantom. The error bars indicate \pm the standard deviation for a series of 10 independent measures.

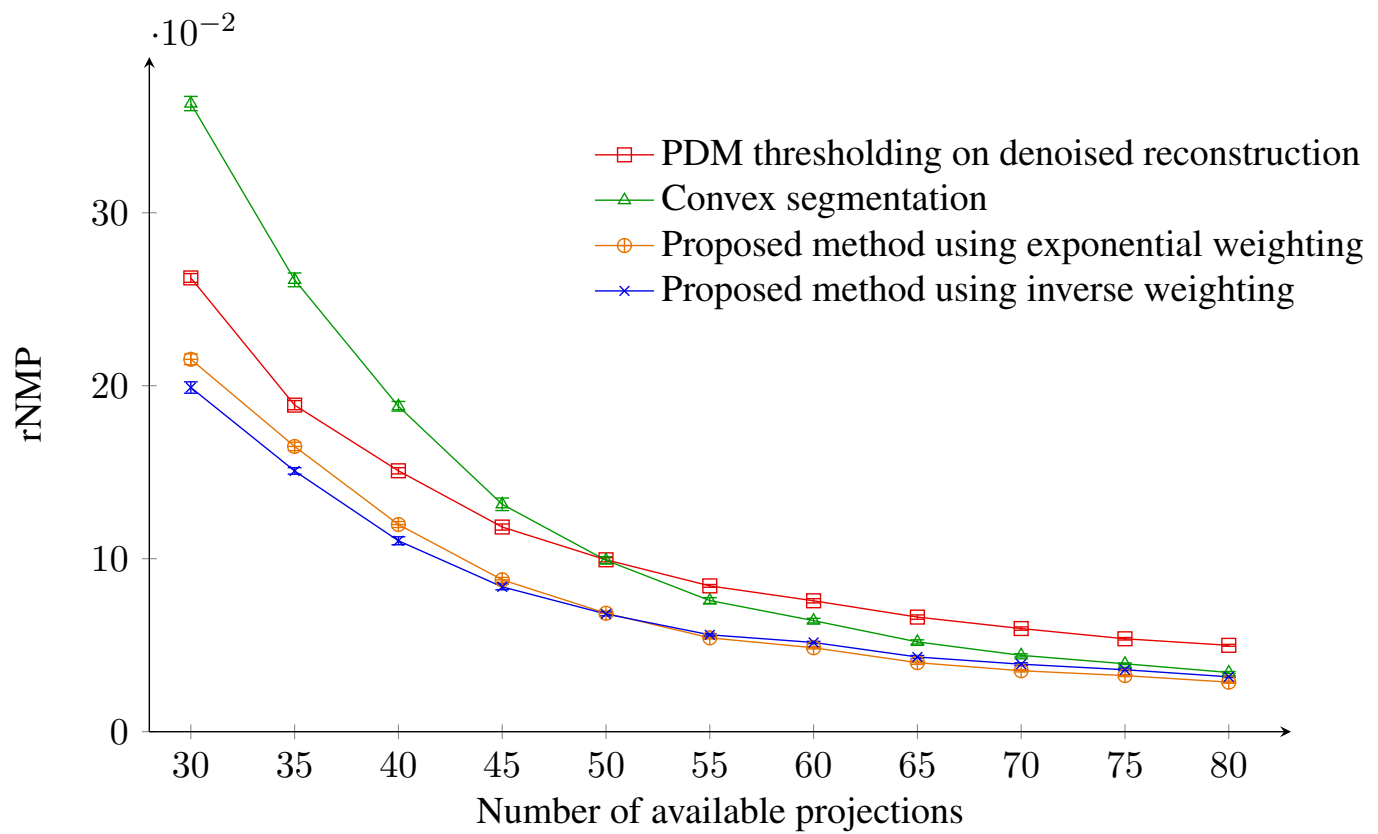

Figure 7: Segmentation error measures (rNMP) for the femur phantom. The error bars indicate \pm the standard deviation for a series of 10 independent measures. 


\subsection{Multilevel segmentation}

Like in the previous section, we used $\lambda=1.5$ for the first convex segmentation step and $\lambda=10^{-4}$ to $10^{-6}$ for the refinement model depending on the chosen weighting function. The phantoms are composed by three different grey levels $(0,1$ and 2$)$.
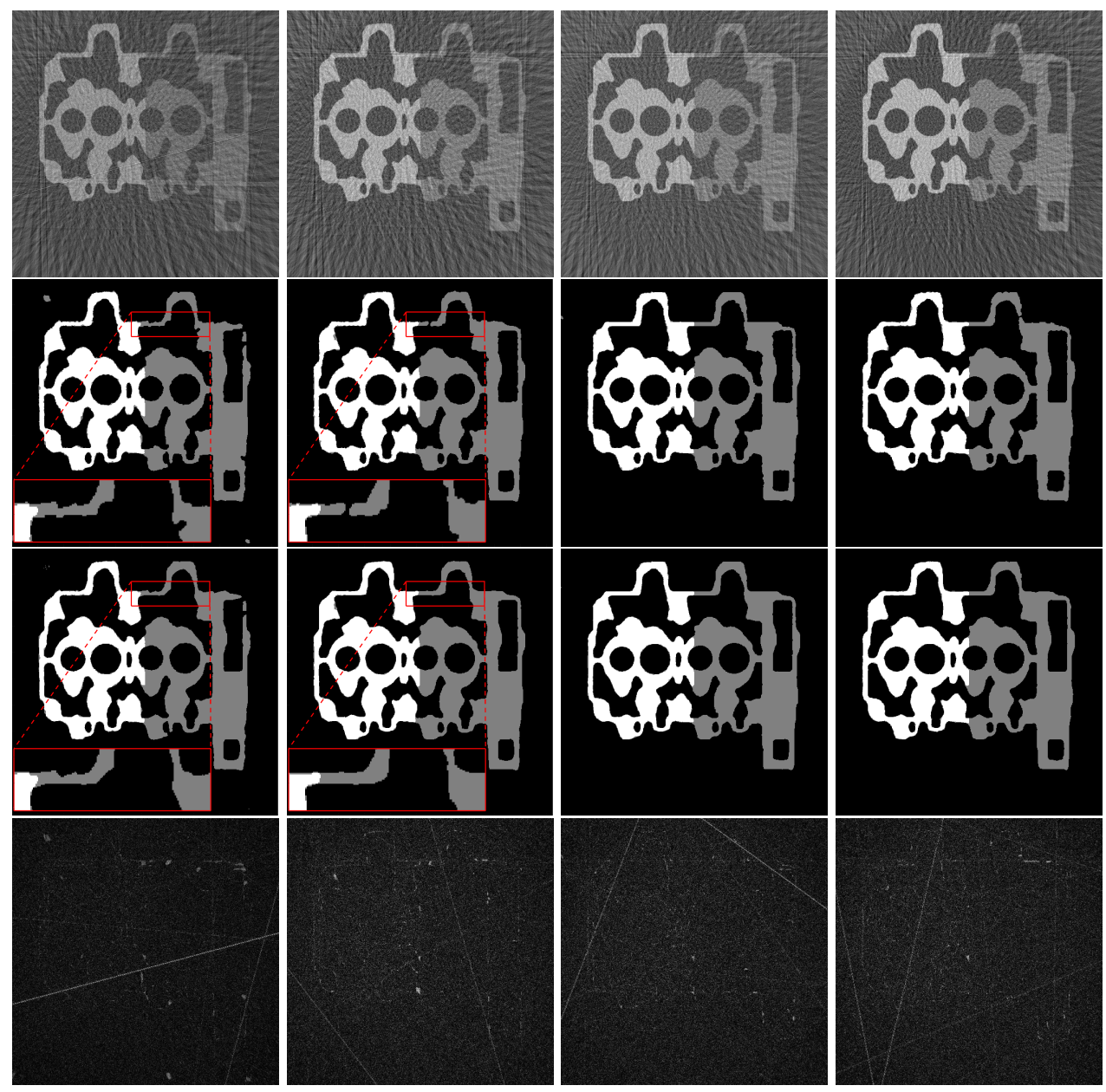

Figure 8: Multi-level cylinder phantom experiment. Each column is obtained by using respectively 45, 50, 55 and 60 projections. First row: Filtered backprojection reconstruction from noisy projections. Second row: Segmentation using convex formulation. Third row: Refined segmentation using our confidence map method. Fourth row: Reconstructed residual error obtained from $2^{\text {nd }}$ row.

Figure 8 shows the result of our segmentation algorithm on the multilevel motor cylinder phantom (fig. 4c). This shows that the algorithm is suitable for segmenting volumes with more than two gray levels. In particular for 45 and 50 projections, the figures show that the refinement process helps significantly in correcting the artifacts in the low confidence regions, like blocky structures and missing 


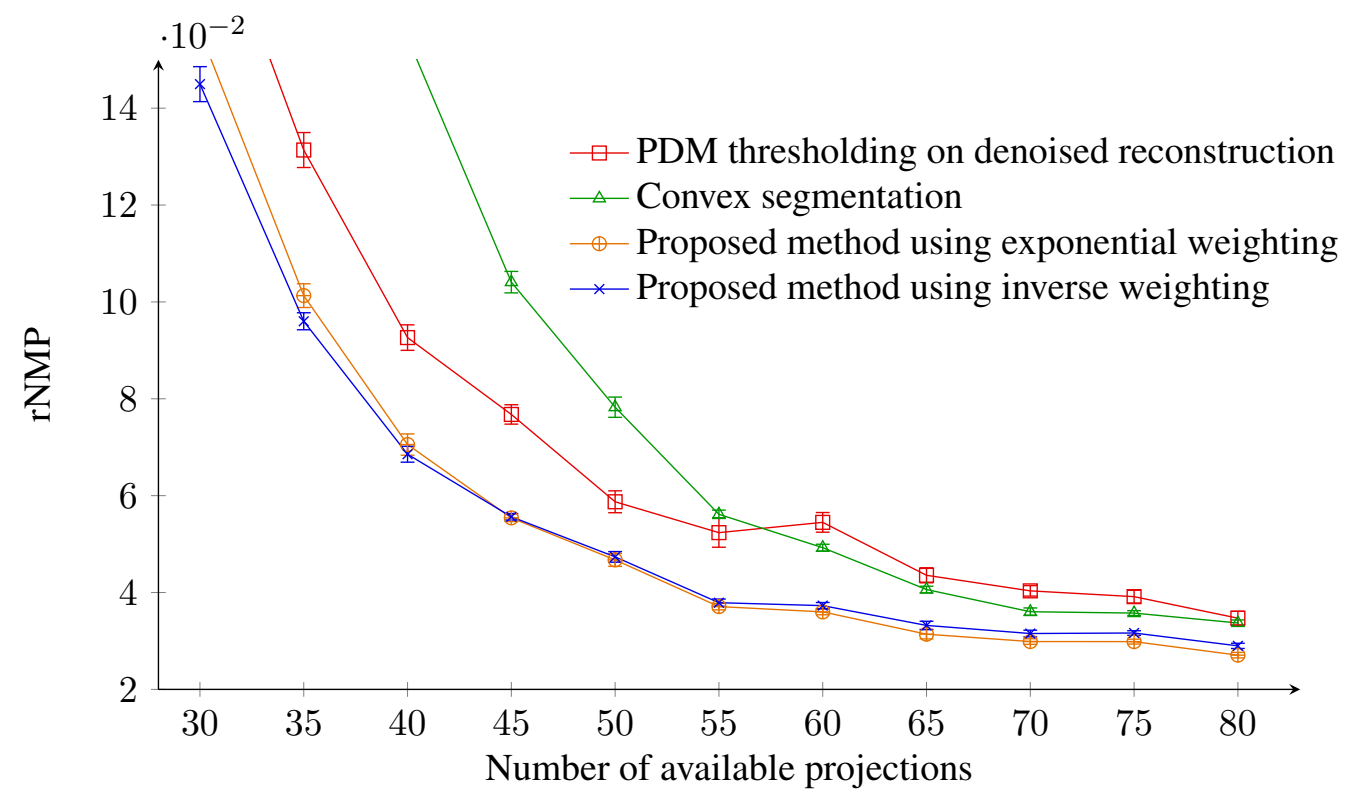

Figure 9: Segmentation error measures (rNMP) for the multilevel cylinder phantom. The error bars indicate \pm the standard deviation for a series of 10 independent measures.

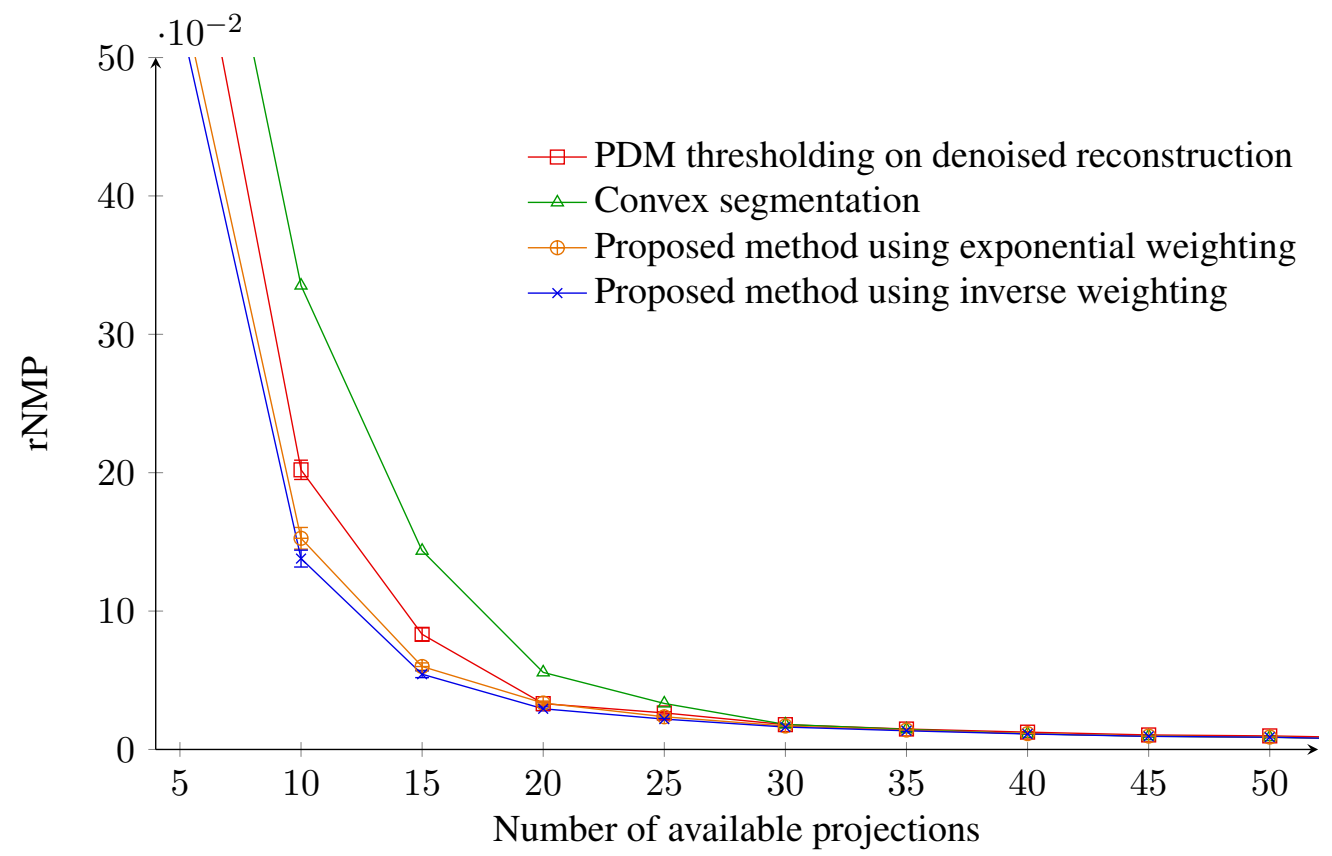

Figure 10: Segmentation error measures (rNMP) for the multilevel shape phantom. The error bars indicate \pm the standard deviation for a series of 10 independent measures. 
edges. We plotted on fig. 9 the pixel error for these figures. They show the superior performance of the proposed method, when compared to the denoising and PDM approach and the initial convex segmentation approach.

Figure 11 shows the three dimensional reconstruction and segmentation from cone-beam acquisition of 60 projections, when using the FDK algorithm both for the initial reconstruction and for the evaluation of the reconstructed residual error. As noted for the two dimensional case in fig. 8, the segmentation is greatly improved by the refinement step of the proposed method.

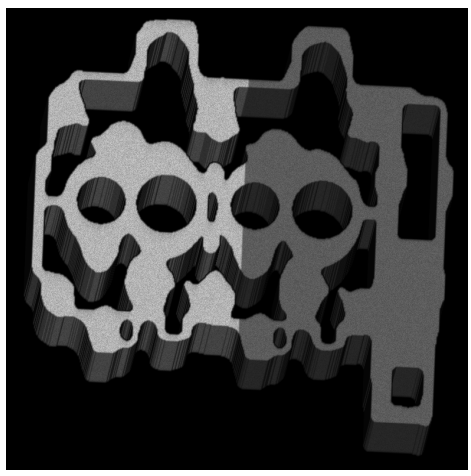

(a)

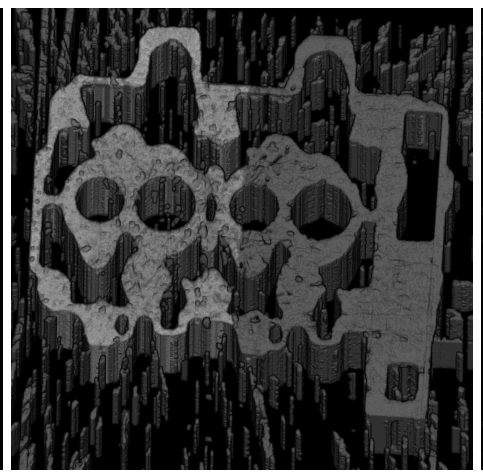

(b)

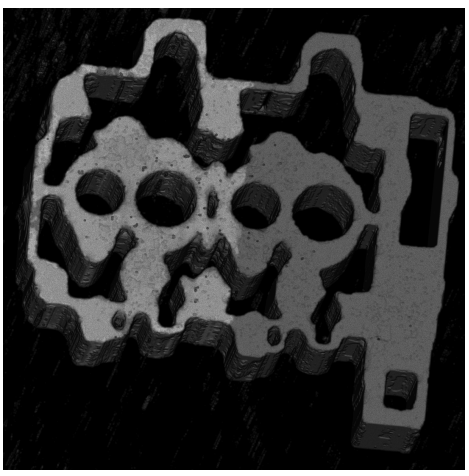

(c)

Figure 11: 3D reconstruction and segmentation of cone-beam data using (a) FDK algorithm, (b) FDK followed by convex segmentation, and (c) FDK followed by our proposed method

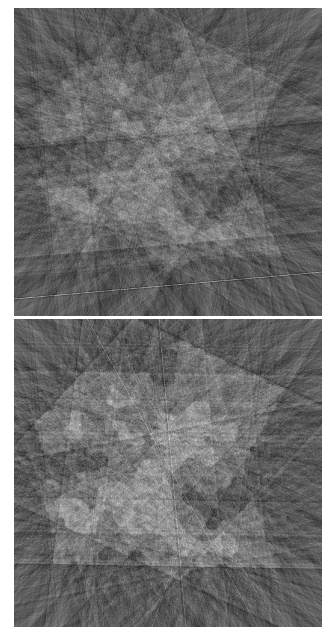

(a) FBP reconstruction

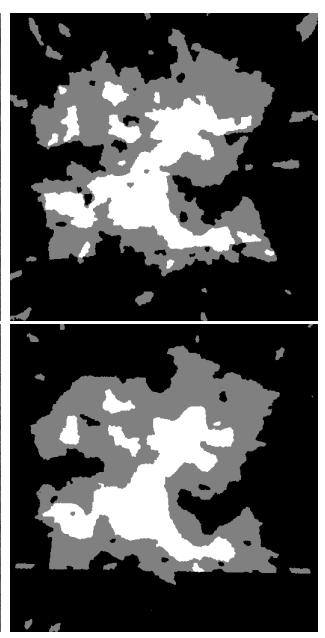

(b) PDM after denoising

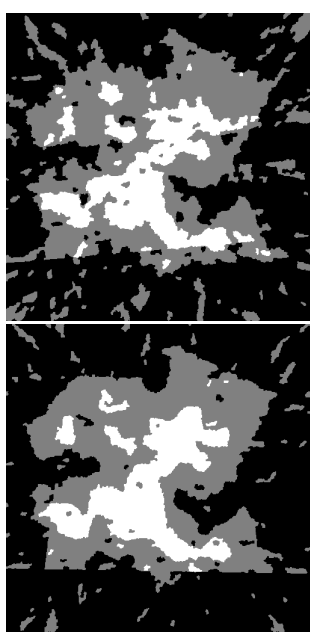

(c) Convex segmentation

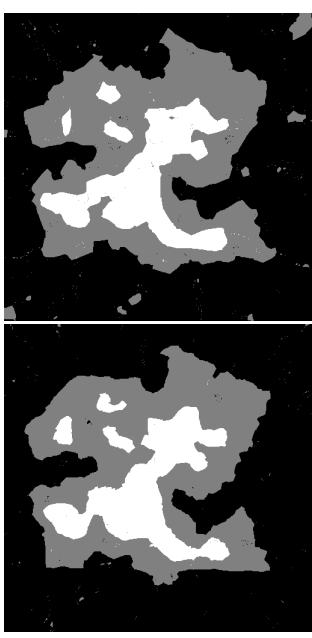

(d) Refined segmentation

Figure 12: Multilevel shape phantom experiment using 10 (first row) and 15 (second row) projections. 
Finally, in fig. 12 we display the segmentation of the multilevel shape phantom for 10 and 15 projections, while in fig. 10 we plotted the performance of the considered approaches with respect to the rNMP for a wide range of projections. This test case shows that the proposed method provides good performance against the other considered methods also for low numbers of projections.

\section{Discussion and conclusions}

In this article, we presented a segmentation scheme that is suitable for real-time tomography applications. Specifically we assume that in such context, little time can be spent in the acquisition of images, in their reconstruction, and ultimately in their segmentation. For this reason we showed that this segmentation scheme can handle reconstructed volumes obtained from moderately low numbers of projections, and from a fast algorithm like FBP. In addition, we assumed that limited time for the acquisition also led to higher levels of noise in the acquired projections, and applied significant levels of noise on top of the acquired projection images.

The results are two-fold. On one side, the core segmentation algorithm is massively parallelizable, it can be run on GPUs, and the memory requirements are not much different from the memory requirements of FBP. This in turn means that it can be run directly on the volumes just reconstructed and still resident in the GPU memory. Subsequently, in the refinement step, the segmented volumes can also be directly forward and back-projected while still being resident on the GPU. As we have seen in section section 2.3, this set of operations guarantees a similar level of performance to the simplest reconstruction methods, and this guarantees a fast execution of the segmentation, in a compatible manner with real-time applications. On the other side, as we saw in section 3, the segmentation performance obtained by this approach is superior to other advanced approaches like thresholding applied to denoised reconstructions. This means that despite being a fast segmentation approach it can also produce robust and high quality results. Moreover, we showed that the segmentation refinement procedure significantly improves the results. The fact that it is actually independent of the initial segmentation method allows it to be flexible and incorporated in other, task-specific processing pipelines.

Another important aspect is that the chosen convex optimization framework comes with the possibility to be easily modified to accommodate for specific features and prior knowledge about the samples. An example is the segmentation of multiple levels, which was addressed in this article. A possible future enhancement instead could be the introduction of dead zones in the gray value ranges, to accommodate for regions that are supposed to be exhibiting intensity gradients or roughness.

\section{Acknowledgements}

HDS wishes to acknowledge financial support by an ERCIM fellowship grant. NV wishes to acknowledge financial support by the European Union's Horizon 2020 research and innovation programme (VOXEL H2020-FETOPEN-2014-2015-RIA GA 665207). 


\section{References}

[1] Aghasi A, Kilmer M, Miller EL. Parametric level set methods for inverse problems, SIAM Journal on Imaging Sciences, 2011;4(2):618-650. doi:10.1137/100800208.

[2] Andersen V, Bjorholm A, Hansen C. Computing segmentations directly from X-ray projection data via parametric deformable curves, Measurement Science and Technology, 2018;29(1):16. doi:10.1088/13616501/aa950e.

[3] Batenburg KJ, Sijbers J. Adaptive thresholding of tomograms by Projection Distance Minimization, Pattern Recognition, 2009;42(10):2297-2305. doi:10.1016/j.patcog.2008.11.027.

[4] Batenburg KJ, Sijbers J. Optimal threshold selection for tomogram segmentation by Projection Distance Minimization, IEEE Transactions on Medical Imaging, 2009;28(5):676-686. doi:10.1109/ TMI.2008.2010437.

[5] Batenburg KJ, Sijbers J. DART: A practical reconstruction algorithm for discrete tomography, IEEE Transactions on Image Processing, 2011;20(9):2542-2553. doi:10.1109/TIP.2011.2131661.

[6] Boykov Y, Jolly MP. Interactive graph cuts for optimal boundary \& region segmentation of objects in N-D images, Proceedings IEEE International Conference on Computer Vision, Vancouver, BC, Canada, 7-14 July 2001. doi:10.1109/ICCV.2001.937505.

[7] Brox T, Weickert J. Level set segmentation with multiple regions, Proceedings IEEE Transactions on Image Processing, October 2006;15(10):3213-3218. doi:10.1109/TIP.2006.877481.

[8] Caselles V, Kimmel R, Sapiro G. Geodesic active contours, International Journal of Computer Vision, 1997;22(1):61-79. doi:10.1023/A:1007979827043.

[9] Chambolle A, Cremers D, Pock T. A convex approach to minimal partitions, SIAM Journal on Imaging Sciences, 2011;5(4):1113-1158. doi:10.1137/110856733. URL https://hal.archives-ouvertes . fr/hal-00630947.

[10] Chambolle A, Pock T. A first-order primal-dual algorithm for convex problems with applications to imaging, Journal of Mathematical Imaging and Vision, 2011;40(1):120-145. doi:10.1007/s10851-010-0251-1.

[11] Chan T, Zhu W. Level set based shape prior segmentation, IEEE Conference on Computer Vision and Pattern Recognition, Beijing, China 17-21 October 2005 pp.1164-1170. doi:10.1109/CVPR.2005.212.

[12] Chan TF, Esedoglu S, Nikolova M. Algorithms for finding global minimizers of image segmentation and denoising models, SIAM Journal on Applied Mathematics, 2006;66(5):1632-1648. doi:10.1137/ 040615286.

[13] Chan TF, Vese LA. Active contours without edges, IEEE Transactions on Image Processing, 2001;10(2): 266-277. doi:10.1109/83.902291.

[14] Couprie C, Grady L, Najman L, Talbot H. Power Watershed: A unifying graph-based optimization framework, IEEE Transactions on Pattern analysis and Machine Intelligence, 2011;33(7):1384-1399. doi:10.1109/TPAMI.2010.200.

[15] Feldkamp LA, Davis LC, Kress JW. Practical cone-beam algorithm, Journal of the Optical Society of America A, 1984;1(6):612-619. URL https://doi .org/10.1364/JOSAA.1.000612.

[16] Grady L. Random walks for image segmentation, IEEE Transactions on Pattern analysis and Machine Intelligence, 2006;28(11):1768-1783. doi:10.1109/TPAMI.2006.233. 
[17] Gregor J, Benson T. Computational analysis and improvement of SIRT, IEEE Transactions on Medical Imaging, 2008;27(7):918-924. doi:10.1109/TMI.2008.923696.

[18] Hansen PC. Analysis of discrete ill-posed problems by means of the L-curve, SIAM Review, 1992; 34(4):561-580. doi:10.1137/1034115.

[19] Kak AC, Slaney M. Principles of computerized tomographic imaging, Classics in Applied Mathematics, Society for Industrial and Applied Mathematics, 2001. ISBN:978-0-89871-494-4. URL https://doi. org/10.1137/1.9780898719277.

[20] Kappes JH, Petra S, Schnör C, Zisler M. TomoGC: Binary tomography by constrained GraphCuts, Pattern Recognition (J. Gall, P. Gehler, B. Leibe, Eds.), vol. 9358, Lecture Notes in Computer Science, 2015 pp. 262-273. doi:10.1007/978-3-319-24947-6_21

[21] Morozov VA. Methods for Solving Incorrectly Posed Problems, Springer Verlag, 1984. ISBN:978-0-38796059-3. doi:10.1007/978-1-4612-5280-1.

[22] Mumford DB, Shah J. Optimal approximations by piecewise smooth functions and associated variational problems, Communications on Pure and Applied Mathematics, 1989;42(5):577-685. URL https:// doi.org/10.1002/cpa.3160420503.

[23] Osher S, Fedkiw RP. Level set methods: An overview and some recent results, Journal of Computational Physics, 2001;169(2):463-502. URL https://doi.org/10.1006/jcph.2000.6636.

[24] Osher S, Sethian JA. Fronts propagating with curvature-dependent speed: algorithms based on HamiltonJacobi formulations, Journal of Computational Physics, 1988;79(1):12-49. URL https://doi.org/ 10.1016/0021-9991(88)90002-2.

[25] Otsu N. A threshold selection method from gray-level histograms, IEEE Transactions on Systems, Man, and Cybernetics, 1979;9(1):62-66. doi:10.1109/TSMC.1979.4310076.

[26] Perona P, Malik J. Scale-space and edge detection using anisotropic diffusion, IEEE Transactions on Pattern Analysis and Machine Intelligence, 1990;12(7):629-639. doi:10.1109/34.56205.

[27] Ramlau R, Ring W. A Mumford-Shah level-set approach for the inversion and segmentation of X-ray tomography data, Journal of Computational Physics, 2007;221(2):539-557. doi:10.1016/j.jcp.2006.06.041.

[28] Roelandts T, Batenburg KJ, den Dekker AJ, Sijbers J. The reconstructed residual error: A novel segmentation evaluation measure for reconstructed images in tomography, Computer Vision and Image Understanding, 2014;126:28-37. doi:10.1016/j.cviu.2014.05.007.

[29] Rousson M, Paragios N. Shape priors for level set representations, European Conference on Computer Vision (A. Heyden, G. Sparr, M. Nielsen, P. Johansen, Eds.), vol. 2351, Lecture Notes in Computer Science, 2002 pp. 78-92. doi:10.1007/3-540-47967-8_6.

[30] Rudin LI, Osher S, Fatemi E. Nonlinear total variation based noise removal algorithms, Physica D: Nonlinear Phenomena, 1992;60(1-4):259-268. URL https : //doi .org/10 .1016/0167-2789(92) 90242-F.

[31] Sezgin M, Sankur B. Survey over image thresholding techniques and quantitative performance evaluation, Journal of Electronic Imaging, 2004;13(1). URL https : //doi .org/10.1117/1.1631315.

[32] Tuysuzoglu A, Karl WC, Stojanovic I, Castañón D, Ünlü, MS. Graph-Cut based discrete-valued image reconstruction, IEEE Transactions on Image Processing, 2015;24(5):1614-1627. doi:10.1109/ TIP.2015.2409568. 
[33] Tuysuzoglu A, Khoo Y, Karl WC. Variable splitting techniques for discrete tomography, Processing IEEE International Conference on Image Processing, 25-28 September 2016 pp. 2329-2340. doi:10.1109/ICIP.2016.7532661.

[34] Van Aarle W, Batenburg KJ, Sijbers J. Optimal threshold selection for segmentation of dense homogeneous objects in tomographic reconstructions, IEEE Transactions on Medical Imaging, 2001;30(4):980-989. doi:10.1109/TMI.2010.2104328.

[35] Van Aarle W, Palenstijn WJ, Cant J, Janssens E, Bleichrodt F, Dabravolski A, Beenhouwer JD, Batenburg KJ, Sijbers J. Fast and flexible X-ray tomography using the ASTRA toolbox, Optics Express, 2016;1(6):25129-25147. doi:10.1364/OE.24.025129.

[36] Vese LA, Chan TF. A multiphase level set framework for image segmentation using the Mumford and Shah model, International Journal of Computer Vision, 2002;50(3):271-293. doi:10.1023/A:1020874308076.

[37] Wang L, Sixou B, Peyrin F. Binary tomography reconstructions with stochastic level-set methods, Medical Physics, 2010;37(5):2329-2340.

[38] Wang L, Sixou B, Peyrin F. Multi-level tomography reconstructions with level-set and TV regularization methods, European Signal Processing Conference, August 2016, Hungary. 2016 pp.1698 - 1702. doi:10.1109/EUSIPCO.2016.7760538.

[39] Weiss P, Blanc-Féraud L, Aubert G. Efficient schemes for Total Variation minimization under constraints in image processing, SIAM Journal on Scientific Computing, 2009;31(3):2047-2080. URL https:// doi.org/10.1137/070696143.

[40] Whitaker RT, Elangovan V. A direct approach to estimating surfaces in tomographic data, Medical Image Analysis, 2002;6(3):235-249. URL https ://doi .org/10.1016/S1361-8415(02)00082-8.

[41] Xu J, Jia Y, Shi Z, Pang K. An improved anisotropic diffusion filter with semi-adaptive threshold for edge preservation, Signal Processing, 2016;119(C):80-91. URL https://doi.org/10.1016/j.sigpro. 2015.07 .017$.

[42] Yoona S, Pineda AR, Fahrig R. Simultaneous segmentation and reconstruction: A level set method approach for limited view computed tomography, Medical Physics, 2010;37(5):2329-2340. doi:10.1118/ 1.3397463.

[43] Zhu SC, Yuille A. Region competition: unifying snakes, region growing, and Bayes/MDL for multiband image segmentation, IEEE Transactions on Pattern Analysis and Machine Intelligence, 1996;18(9):884900. doi: $10.1109 / 34.537343$.

[44] Zhuge X, Palenstijn WJ, Batenburg KJ. TVR-DART: A more robust algorithm for discrete tomography from limited projection data with automated gray value estimation, IEEE Transactions on Image Processing, 2016;25(1):455-468. doi:10.1109/TIP.2015.2504869. 